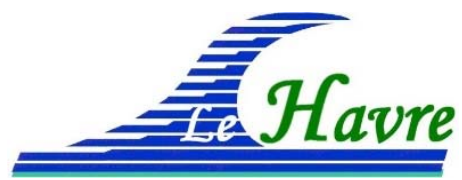

\title{
A new model of shoaling and breaking waves - Wave trains and two-dimensional applications
}

\author{
Arnaud DURAN ${ }^{1}$, Benoît FABREGES ${ }^{2}$, \\ Maria KAZAKOVA ${ }^{2}$, Gaël Loïc RICHARD ${ }^{3}$
}

1. Université Claude Bernard Lyon 1, Institut Camille Jordan (ICJ) - UMR 5208. 43 boulevard du 11 novembre 1918, 69622 Villeurbanne cedex, France. arnaud.duran@univ-lyon1.fr ; benoit.fabreges@univ-lyon1.fr

2. Laboratoire de Mathématiques de l'INSA de Rouen (LMI) - EA 3226 - FR CNRS 3335. 685 Avenue de l'Université, 76800 Saint-Étienne-du-Rouvray, France. maria.kazakova@insa-rouen.fr

3. Université Grenoble Alpes, IRSTEA, ETNA, 38000 Grenoble, France. gael.richard@univ-grenoble-alpes.fr

\section{Résumé :}

La propagation des vagues dans les zones côtières implique des mécanismes complexes, représentant des enjeux de modélisation et numériques considérables. Si la plupart des processus non-linéaires sont généralement capturés par des modèles de type Boussinesq, ces équations conservent l'énergie et sont donc intrinsèquement inaptes à décrire les mécanismes dissipatifs, tels que ceux associés au déferlement des vagues. Pour gérer ce phénomène, nous introduisons un nouveau modèle dispersif fortement non-linéaire capable de prendre en compte les effets turbulents sous-jacents. L'approche est caractérisée par la présence d'une nouvelle variable basée sur la variation verticale de la vitesse, appelée enstrophie, modélisant l'énergie turbulente. Le modèle proposé présente une structure similaire aux équations de Green-Naghdi $(\mathrm{GN})$ et peut donc être intégré numériquement sur la base de toute méthode existante pour ces équations. Dans le prolongement de travaux récents, nous considérons une discrétisation de type Galerkin discontinu du système, basée sur un découplage entre les parties hyperboliques et nonhydrostatiques. Des validations numériques 1D et 2D impliquant la propagation de vagues déferlantes sur des topographies non triviales sont proposées. En particulier, les comparaisons avec les données expérimentales confirment l'efficacité de la stratégie, mettant en évidence l'enstrophie comme un outil robuste et fiable pour la détection et la description des vagues déferlantes.

Mots-clés : Océanographie côtière, Déferlement, Turbulence, Dispersion.

\section{Introduction}

Depuis plusieurs décennies, la modélisation mathématique et numérique en océanographie, représente un secteur d'activité intensif au sein la communauté scientifique. Devant les enjeux climatiques actuels (fréquence et intensité des évènements 


\section{Thème 1 - Hydrodynamique côtière}

extrêmes, montée des eaux, érosion, production d'énergie marine,...), la conception de modèles de prévision fiables et efficaces dédiés à l'océanographie côtière est devenue une nécessité de premier plan. En dépit de constants progrès techniques, la résolution directe des équations de Navier-Stokes reste toujours hors de portée d'un point de vue opérationnel. Dans ce contexte, l'intérêt se porte sur des modèles simplifiés, moins coûteux numériquement, avec notamment les modèles d'écoulement à surface libre. Concernant le mécanisme de déferlement, l'un des principaux enjeux est de réussir à décrire à la fois la dispersion (dominante avant le déferlement) et la dissipation, dominante dans la zone de déferlement. Le caractère conservatif des modèles dispersifs de type Boussinesq ne permettant pas de décrire la dissipation, il est nécessaire d'introduire des méthodes spécifiques pour gérer ce phénomène. A l'heure actuelle, dans le contexte de modèles moyennés sur la verticale, la plupart des méthodes existantes sont classifiées en deux catégories. La première stratégie consiste à activer un terme de viscosité artificielle dans les équations au niveau de la zone de déferlement pour modéliser la dissipation. Nous renvoyons à CHEN et al. (2000), KIRBY et al. (1998), ou bien à NWOGU (1996) ou ZHANG et al. (2014) où la viscosité est déterminée à partir d'une énergie turbulente à travers une équation de transport. Une seconde approche, généralement appelée méthode hybride (ou switching en anglais) consiste à traiter les vagues déferlantes comme des chocs dans les équations de SaintVenant en supprimant les termes dispersifs à partir du point de déferlement. Depuis une dizaine d'années, ce type d'approche a été utilisé dans de nombreux contextes. De manière générale, chacune de ces approches requiert la calibration d'un jeu de paramètres empiriques, et doit être couplée à un critère de détection pour activer ou désactiver le mécanisme de dissipation. Ces paramètres peuvent varier en fonction du type de déferlement étudié, ou bien encore des choix de discrétisation, compromettant le caractère prédictif de la méthode. Sur ces aspects, une analyse détaillée est proposée dans l'étude récente KAZOLEA \& RICCHIUTO (2018).

Plus récemment, un autre type d'approche a commencé à se développer, prenant en compte les variations verticales du profil de vitesse à travers l'ajout une troisième variable dans le modèle TESHUKOV (2007). Adjointes à un mécanisme de dissipation, ces méthodes permettent une excellente description des roll-waves et des ressauts hydrauliques (RICHARD \& GAVRILYUK, 2012, 2013). La fermeture du modèle est obtenue par une hypothèse d'écoulement faiblement cisaillé. La prise en compte des effets dispersifs a ensuite été proposée par CASTRO \& LANNES (2014), RICHARD et GAVRILYUK (2015), travaux suivis par une première application au déferlement GAVRILYUK et al. (2016) dans le cadre d'un modèle à deux couches. Une première proposition permettant d'inclure à la fois la dispersion et la dissipation dans un modèle à une couche a récemment été développée par KAZAKOVA \& RICHARD (2019), et étendue par RICHARD et al. (2019) en dimension 2. La turbulence de grande échelle est explicitement résolue tandis que la turbulence de petite échelle est modélisée par une 


\section{XVİ̀mes Journées Nationales Génie Côtier - Génie Civil \\ Le Havre 2020}

hypothèse de viscosité turbulente. Les termes dispersifs étant traités de manière similaire aux équations GN, le modèle obtenu peut se traiter numériquement sur la base de techniques existantes pour ces équations. Il en résulte une approche permettant de décrire le déferlement de manière robuste et prédictive dans une grande variété de contextes. Ce papier fait état des principaux résultats obtenus en $1 \mathrm{D}$ et $2 \mathrm{D}$ dans le cas des ondes solitaires, et de la récente extension aux trains d'ondes.

\section{Equations du modèle}

Dénotant $h_{0}^{*}$ une profondeur de référence et $L$ une longueur caractéristique des variations horizontales de l'écoulement, le modèle est dérivé sous hypothèse d'écoulements à faible profondeur: $\mu=h_{0}^{*} / L \ll 1$. Dans ce contexte, l'obtention du modèle repose sur l'intégration des équations LES (Large Eddy Simulation) selon la verticale. La vitesse horizontale filtrée est décomposée en une vitesse moyenne sur la profondeur et un écart à cette vitesse moyenne, représentant la turbulence de grande échelle et les effets de cisaillement: $\bar{u}(\boldsymbol{x}, z, t)=U(\boldsymbol{x}, t)+u^{\prime}(\boldsymbol{x}, z, t)$. La turbulence de grande échelle, qui contient l'essentiel de l'énergie associée au mécanisme de déferlement, est ainsi explicitement résolue en introduisant la quantité suivante, appelée enstrophie:

$\varphi=\frac{1}{h^{3}} \int_{b}^{b+h}\left(u^{\prime}\right)^{2} d z$

où $h$ désigne la hauteur d'eau et $b$ une paramétrisation de la topographie (figure 1). Comme dans TESHUKOV (2007), l'écoulement est supposé faiblement turbulent et faiblement cisaillé. La fermeture du modèle est obtenue en modélisant la turbulence de petite échelle par une hypothèse de viscosité turbulente. Au final, en $1 \mathrm{D}$, le modèle obtenu est constitué des trois équations suivantes:

$\frac{\partial h}{\partial t}+\frac{\partial h U}{\partial x}=0$

$\frac{\partial h U}{\partial t}+\frac{\partial}{\partial x}\left(h U^{2}+h^{3} \varphi+\frac{g h^{2}}{2}+\frac{h^{2} \ddot{h}}{3}+\Pi^{\prime}\right)=\frac{\partial}{\partial x}\left(\frac{4}{R} h^{3} \sqrt{\varphi} \frac{\partial U}{\partial x}\right)-g h \frac{\partial b}{\partial x}-f^{\prime}$,

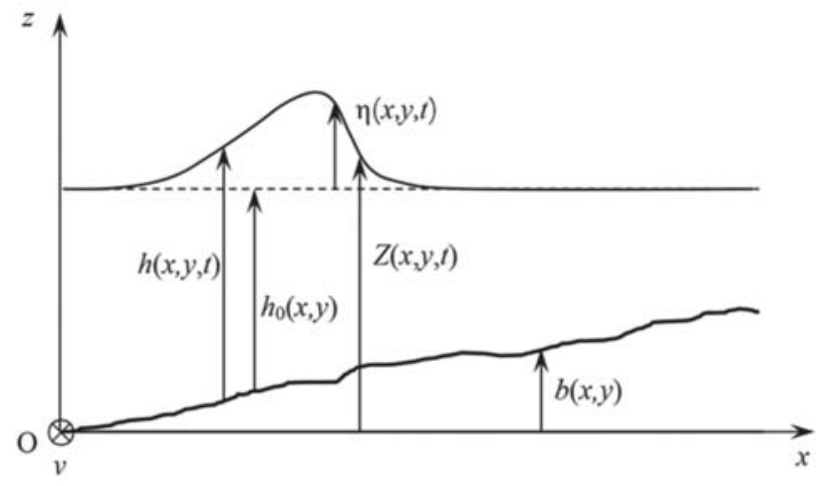

Figure 1. Notations. 


\section{Thème 1 - Hydrodynamique côtière}

où $\ddot{h}$ désigne la dérivée matérielle seconde de $h$, et $\Pi^{\prime}$ et $f^{\prime}$ sont des termes d'ordre $\mathcal{O}\left(\mu^{2}\right)$ liés au fond variable:

$\Pi^{\prime}=\frac{h^{2}}{2} \frac{D}{D t}\left(U \frac{\partial b}{\partial x}\right), f^{\prime}=h \frac{\partial b}{\partial x}\left[\frac{\ddot{h}}{2}+\frac{D}{D t}\left(U \frac{\partial b}{\partial x}\right)\right]$

La dérivée matérielle est $D / D t=\partial / \partial t+U \partial / \partial x$. L'équation sur l'enstrophie est donnée par:

$\frac{\partial h \varphi}{\partial t}+\frac{\partial h U \varphi}{\partial x}=\frac{8 v_{T}}{h}\left(\frac{\partial U}{\partial x}\right)^{2}-C_{r} h \varphi^{3 / 2}+\frac{\partial}{\partial x}\left(2 \frac{h^{3} \sqrt{\varphi}}{\sigma R} \frac{\partial \varphi}{\partial x}\right)$

où $v_{T}=h^{2} \sqrt{\varphi} / R$ désigne la viscosité turbulente, la quantité $R$ pouvant être vue comme un nombre de Reynolds sans dimension. La constante $\sigma>0$ gouverne un terme de diffusion sur l'enstrophie pouvant être négligé dans le cas des ondes solitaires (formellement $\sigma=+\infty$ ). Dans le cas des trains d'ondes, ce terme a un effet régularisant au niveau du point de déferlement. En pratique, nous prenons $\sigma=1$ mais toute autre valeur de cet ordre peut être envisagée sans modifier significativement la solution. En dimension deux, $\varphi$ est un tenseur anisotrope et donne lieu à trois équations supplémentaires au lieu d'une. Notons cependant que l'équation sur l'enstrophie ne contient aucun terme dispersif ni aucun terme associé au fond variable, et ne représente à ce titre aucune difficulté particulière d'un point de vue numérique. Comme dans les équations GN, la difficulté réside dans l'équation de quantité de mouvement qui contient notamment des dérivées croisées en temps et en espace, à travers la dérivée matérielle. Des méthodes récentes dédiées aux équations GN, basées sur des modèles asymptotiquement équivalents (BONNETON et al. 2012 ; LANNES \& MARCHE, 2015) permettent de reformuler le problème d'une manière plus appropriée, tout en améliorant les propriétés dispersives. Ces méthodes ont récemment été étendues aux modèles $1 \mathrm{D}$ et 2D avec enstrophie, dans un environnement de type Galerkin discontinu basé sur les travaux de DURAN \& MARCHE (2017).

\section{Validation du modèle}

Dans les travaux de KAZAKOVA \& RICHARD (2019), il a été établi que pour de faibles non-linéarités (c'est-à-dire $\delta<0,05$, où $\delta=a / h_{0}^{*}$ avec $a$ l'amplitude de la vague), les résultats obtenus sont en bon accord avec l'expérience et l'enstrophie est un indicateur fiable de début de déferlement. La valeur $C_{r}=0,48$ semble être universelle, et les résultats ne se sont pas révélés sensibles au choix du second paramètre $R$ du modèle (le choix $5 \leq R \leq 10$ fournit de très bons résultats). Cependant, pour des non linéarités plus fortes, l'activation de l'enstrophie dès le début des calculs peut avoir un impact sur l'amplitude des vagues, et ce même en dehors des zones de déferlement. Une solution consiste à activer les termes visqueux (associés à la création d'enstrophie) uniquement au niveau de ces zones. Des critères classiques peuvent être utilisés, mais l'enstrophie ellemême, associée par construction à l'énergie turbulente, peut être utilisée comme critère de détection. Ceci mène à l'introduction d'une nouvelle quantité, appelée enstrophie 


\section{XVI'èmes Journées Nationales Génie Côtier - Génie Civil \\ Le Havre 2020}

virtuelle, qui évalue la quantité d'enstrophie que le modèle est capable de produire. D'un point de vue pratique, cette quantité est calculée exactement comme l'enstrophie dans tout le domaine de calcul, mais sans rétroaction sur les autres variables. Les zones de déferlement peuvent alors être identifiées par une production brutale d'enstrophie virtuelle. Le seuil de détection numérique est donné par (nous référons aux travaux de KAZAKOVA \& RICHARD (2019) pour plus de détails) :

$\psi_{0}=\frac{g}{h_{0}^{*}}\left(0.1+\frac{0,031}{\delta}\right)$

Dans ce contexte, le nombre de Reynolds adimensionné est donné par $R=0,85+60$ s, $S$ étant une estimation locale de la pente, et le terme de viscosité $v_{T}$ est nul dans l'équation (5) dans les zones où $\psi \leq \psi_{0}$.

Les expériences de TING \& KIRBY (1994) permettent d'étudier le comportement du modèle pour deux types de déferlement (glissant et plongeant). La simulation implique un train de vague sinusoïdal se propageant sur une pente 1/35. Le déferlement glissant correspond à une amplitude $a=12,5 \mathrm{~cm}$ et une période $T=2 \mathrm{~s}$, et $a=12,8 \mathrm{~cm}, T=$ $5 s$ dans le cas plongeant. La figure 2 permet de comparer les solutions obtenues pour ces deux configurations, à travers l'évolution temporelle de l'énergie turbulente $h^{2} \varphi / 2 g h$ et de la vitesse moyenne au voisinage du point de déferlement. On peut notamment observer que le déferlement glissant se caractérise par le fait que l'énergie turbulente n'a pas le temps de se dissiper totalement entre deux épisodes de déferlement. Il en résulte que le courant de retour induit un transport d'énergie turbulente vers le large. Pour le déferlement plongeant, l'énergie turbulente a le temps de se dissiper, de sorte que le transport d'énergie turbulente est dirigé vers la côte. Ces résultats sont tout à fait conformes aux observations expérimentales, de même que l'évolution temporelle de la surface libre. A noter que, contrairement aux approches de type switching, l'approche s'est révélée être peu sensible aux paramètres de discrétisation et permet la convergence en maillage.

Les études expérimentales de BEJI \& BATTJES (1993) ont permis de valider le modèle pour différents types de trains de vagues se propageant et déferlant sur une topographie non triviale. Ces expériences ont d'abord été utilisées avec succès par RICHARD et al., 2019 dans le cas de vagues régulières, mais la stratégie s'avère efficace dans le cas de vagues irrégulières. Nous illustrons ici les résultats obtenus avec un profil de vagues aléatoires de type JONSWAP correspondant à une fréquence caractéristique $h=0,4 \mathrm{~Hz}$. La figure 3 montre l'évolution temporelle des profils de surface libre au niveau de différentes sondes disposées autour du point de déferlement, pour différentes valeurs de $R$. On peut observer une très bonne correspondance avec les résultats expérimentaux.

Les expériences de SWIGLER (2009) ont été utilisées pour valider le modèle 2d. Il s'agit d'étudier le déferlement d'une onde solitaire sur une plage comprenant un récif conique. La simulation a été réalisée sur un maillage non structuré de 200000 mailles, raffiné au niveau du récif. Nous proposons en figure 4 des profils de la surface libre à différents temps de la propagation. La trace du tenseur d'enstrophie, illustrée en rouge, permet de 


\section{Thème 1 - Hydrodynamique côtière}

marquer clairement le début de l'épisode de déferlement à l'arrivée de la vague sur le récif. Les structures turbulentes se propagent ensuite latéralement pour former un front déferlant sur toute la largeur du bassin. L'évolution temporelle de la surface libre et des composantes de la vitesses à différentes sondes sont en bon accord avec l'expérience.
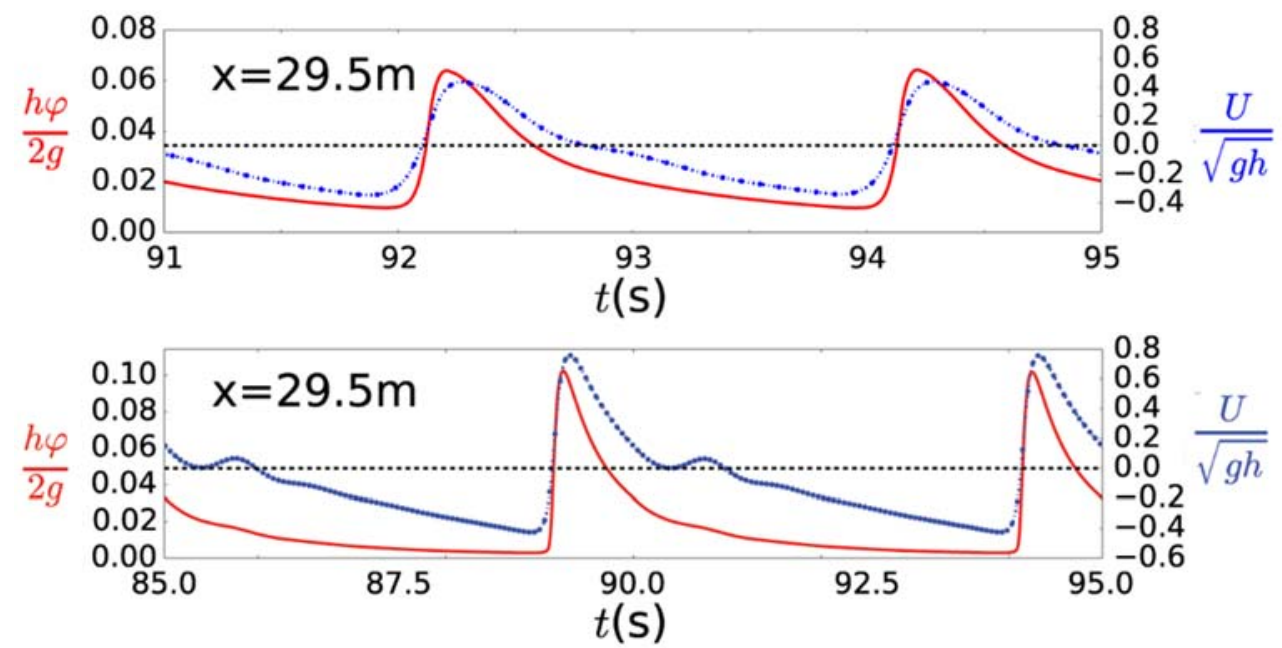

Figure 2. Expériences de TING \& KIRBY (1994). Evolution temporelle de l'énergie turbulente adimensionnée (rouge) et de la vitesse moyenne (bleu) dans le cas de déferlements glissant (haut) et plongeant (bas).

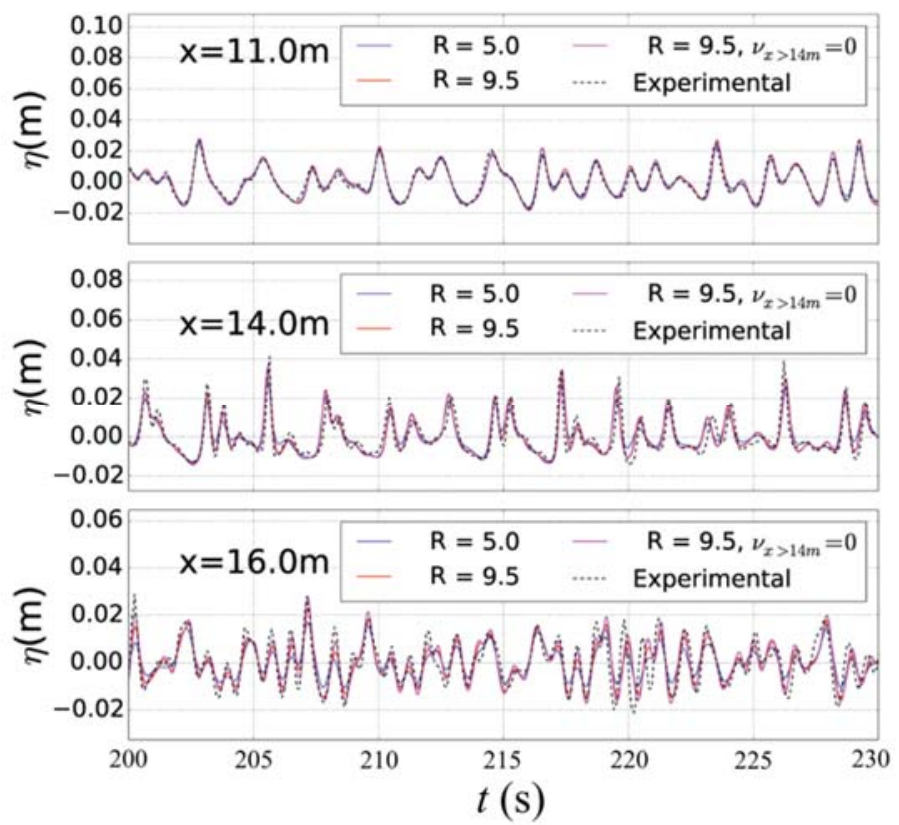

Figure 3. Expérience de BEJI \& BATTJES (1993) - Déferlement de vagues irrégulières. Evolution temporelle de la surface libre. 


\section{XVI'̀mes Journées Nationales Génie Côtier - Génie Civil}

Le Havre 2020
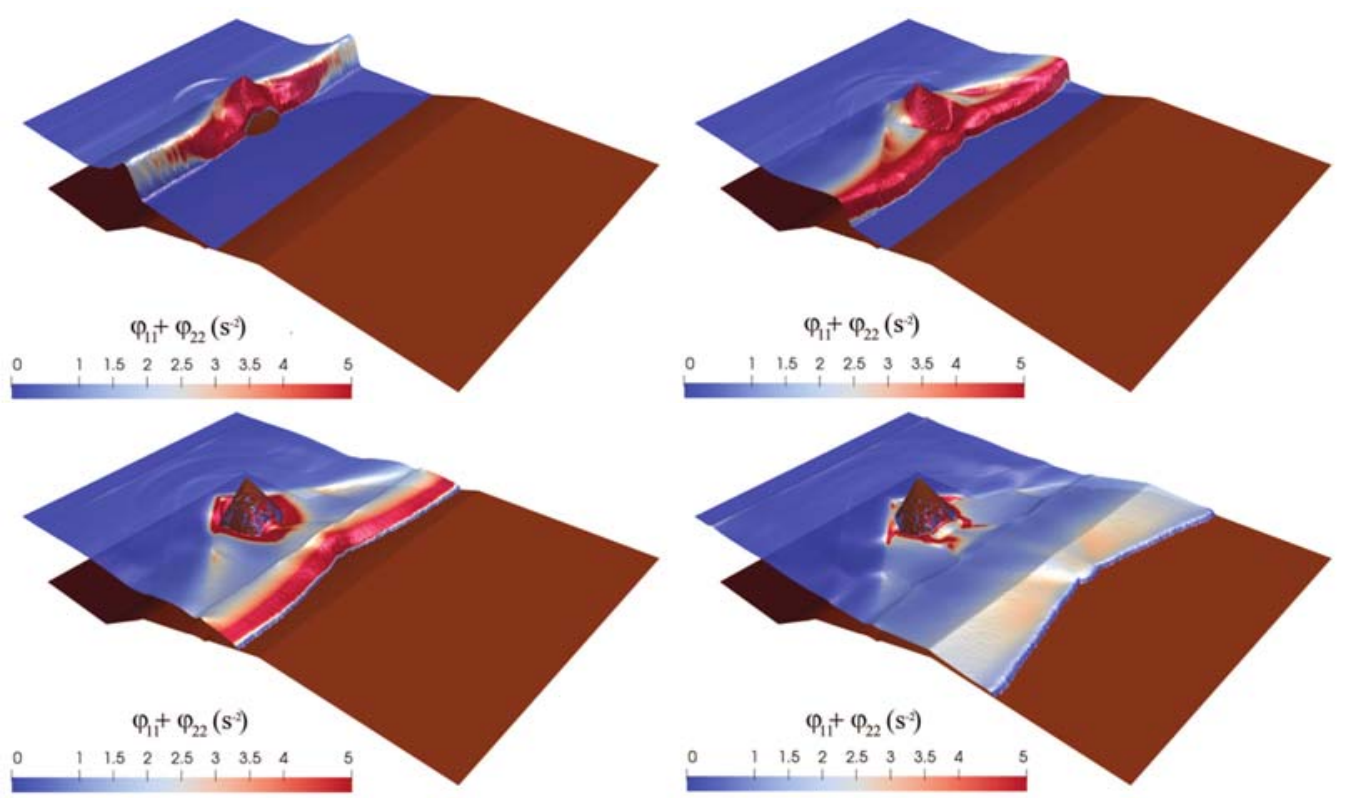

Figure 4. Expériences de SWIGLER, 2009 - Profils de surface libre à différentes étapes du déferlement.

\section{Conclusions}

Nous proposons une nouvelle approche pour décrire le déferlement des vagues, basée sur un modèle turbulent moyenné sur la profondeur. Les résultats présentés étendent les travaux de KAZAKOVA \& RICHARD (2019) au cas des trains d'ondes et en 2D. La turbulence de grande échelle associée au mécanisme de déferlement est explicitement résolue grâce à l'introduction d'une nouvelle variable, appelée enstrophie. Le modèle présente la même structure que les équations de GN, auxquelles doivent adjointes une équation de transport (trois en dimension deux). Ces équations supplémentaires ne représentent pas de difficultés numériques particulières et le modèle peut être traité avec les mêmes techniques que celles utilisées pour le modèle GN. A ce titre, l'approche de type Galerkin Discontinu de DURAN \& MARCHE (2017) a été utilisée, couplée aux méthodes de BONNETON et al. (2012) et LANNES \& MARCHE (2015) pour améliorer les propriétés dispersives et les performances en temps de calcul. En gardant les mêmes paramètres que dans le cas d'une onde solitaire, le modèle permet de décrire le déferlement de trains d'ondes, même dans le cas de vagues irrégulières, et s'étend à des configurations 2D impliquant des fronts secs et des topographies complexes.

\section{Références bibliographiques}

BEJI S., BATTJES J.A. (1993). Experimental investigations of wave propagation over a bar. Coast. Eng., Vol. 19, pp 151-162. https://doi.org/10.1016/0378-3839(93)90022-Z 


\section{Thème 1 - Hydrodynamique côtière}

BONNETON P., CHAZEL F., LANNES D., MARCHE F., TISSIER M. (2012). A splitting approach for the fully nonlinear and weakly dispersive Green-Naghdi model. J. Comput. Phys., Vol. 230, pp 1479-1498. https://doi.org/10.1016/j.jcp.2010.11.015

CASTRO A., LANNES D. (2014). Fully nonlinear long-wave models in the presence of vorticity. Journal of Fluid Mechanics, Vol. 759, pp 642-675. https://doi.org/10.1017/jfm.2014.593 CHEN Q., KIRBY J.T., DALRYMPLE R.A., KENNEDY A.B., CHAWLA A. (2000). Boussinesq modeling of wave transformation, breaking and runup. II: 2D. J. Waterw. Port Coast., Vol. 126, pp 48-56. https://doi.org/10.1061/(ASCE)0733-950X(2000)126:1(48) DURAN A., MARCHE F. (2017). A discontinuous Galerkin method for a new class of Green-Naghdi equations on simplicial unstructured meshes. Appl. Math. Model., Vol. 45, pp 840-864. https://doi.org/10.1016/j.apm.2017.01.030

GAVRILYUK S., LIAPIDEVSKII V.Y., CHESNOKOV A.A. (2016). Spilling breakers in shallow water: applications to Favre waves and to the shoaling and breaking of solitary waves. Journal of Fluid Mechanics, Vol. 808, pp 441-468. https://doi.org/10.1017/jfm.2016.662

KAZAKOVA M., RICHARD G.L. (2019). A new model of shoaling and breaking waves: One-dimensional solitary wave on a mild sloping beach. Journal of Fluid Mechanics, Vol. 862, pp 552-591. https://doi.org/10.1017/jfm.2018.947

KAZOLEA M., RICCHIUTO M. (2018). On wave breaking for Boussinesq-type models. Ocean Model., Vol. 123, pp 16-39. https://doi.org/10.1016/j.ocemod.2018.01.003

KIRBY J.T., WEI G., CHEN Q., KENNEDY A.B., DALRYMPLE R.A. (1998). FUNWAVE 1.0. Fully nonlinear Boussinesq wave model. Technical Report CACR-9806, Center for Applied Coastal Research, Department of Civil and Environmental Engineering, University of Delaware.

LANNES D., MARCHE F. (2015). A new class of fully nonlinear and weakly dispersive Green-Naghdi models for efficient 2D simulation. J. C. Phys., Vol. 282, pp 238-268. https://doi.org/10.1016/j.jcp.2014.11.016

NWOGU O.K. (1996). Numerical prediction of breaking waves and currents with a Boussinesq model. Coastal Engineering, Vol. 25, pp 4807-4820.

RICHARD G.L., GAVRILYUK S. (2012). A new model of roll waves: comparison with Brock’s experiments. J. Fluid Mech., Vol. 698, pp 374-405. https://doi.org/10.1017/jfm.2012.96 RICHARD G.L., GAVRILYUK S. (2013). The classical hydraulic jump in a model of shear shallow-water flows. J. Fluid Mech., Vol. 725, pp 492-521. https://doi.org/10.1017/jfm.2013.174

RICHARD G.L., GAVRILYUK S. (2015). Modelling turbulence generation in solitary waves on shear shallow water flows. J. Fluid Mech., Vol. 773, pp 49-74. https://doi.org/10.1017/jfm.2015.236

RICHARD G.L., DURAN A., FABREGES, B. (2019). A high-order adaptive timestepping TVD solver for Boussinesq modeling of breaking waves and coastal inundation. J. Fluid Mech., Vol. 867, pp 146-194. https://doi.org/10.1017/jfm.2019.125 


\section{XVİ̀mes Journées Nationales Génie Côtier - Génie Civil \\ Le Havre 2020}

SWIGLER D.T. (2009). Laboratory study investigating the three-dimensional turbulence and kinematic properties associated with a breaking solitary wave. PHD Thesis, Texas A\&M University.

TESHUKOV V.M. (2007). Gas-dynamics analogy for vortex free-boundary flows. J. Applied Mechanics and Technical Physics, Vol. 48, pp 303-309. https://doi.org/10.1007/s10808007-0039-2

TING F.C.K., KIRBY J.T. (1994). Observation of undertow and turbulence in a laboratory surf zone. Coast. Eng., Vol. 24, pp 51-80. https://doi.org/10.1016/0378-3839(94)90026-4 ZHANG Y., KENNEDY A.B., DONAHUE A.S., WESTERINK J.J., PANDA N., DAWSON C. (2014). Rotational surf zone modeling for $O\left(\mu^{4}\right)$ Boussinesq-GreenNaghdi systems. Ocean Model., Vol. 79, pp 43-53. https://doi.org/10.1016/j.ocemod.2014.04.001 
Thème 1 - Hydrodynamique côtière 